\title{
Neoplastic severe central airways obstruction, interventional bronchoscopy: A decision-making analysis
}

\author{
Venceslau Hespanhol, $\mathrm{PhD},{ }^{\mathrm{a}, \mathrm{b}}$ Adriana Magalhães, $\mathrm{MD},{ }^{\mathrm{a}}$ and Agostinho Marques, $\mathrm{PhD}^{\mathrm{a}, \mathrm{b}}$
}

\begin{abstract}
Objective: Cancer can involve the airways, causing various degrees of obstruction. Usually, after days or months of mild to moderate undervalued symptoms, severe dyspnea arises abruptly, imposing an immediate attempt to restore the airflow regardless of the etiology. This study focuses on the development of a predictive preintervention model that is useful when deciding whether to perform therapeutic interventional bronchoscopy in patients with severe central airway obstruction.
\end{abstract}

\begin{abstract}
Methods: A total of 804 patients who underwent rigid bronchoscopy under general anesthesia to treat severe neoplastic central airway obstruction from 1990 to 2009 were studied. Electronic records for patients who underwent bronchoscopy were analyzed. The patients were primarily male $(\mathrm{n}=618,76.9 \%)$ and the median age was 62 years. Lung cancer was the most frequent cause of neoplastic airway obstruction $(n=645,81.65 \%)$. An estimate of the probability of individual endoscopic success was made.
\end{abstract}

Results: Of the 804 patients with severe neoplastic airway obstruction, 681 (84.7\%) achieved luminal clearance, and the procedure was considered an endoscopic success. Tracheal involvement (rate ratio, 1.21; range, 1.16-1.27) endoluminal mass (rate ratio, 1.13; range, 1.06-1.12), and extrinsic compression (rate ratio, 1.17; 1.11-1.17) were associated significantly with a favorable endoscopic outcome. Tumor location and any kind of mucosal infiltration were the main determinants of the predictive preoperative model of intervention success.

Conclusions: Endoscopic characteristics and location of the neoplastic lesions are the major determinants of patients' endoscopic outcome. The preintervention model adds to the clinical evaluation an important contribution to the decision-making process on performing therapeutic interventional bronchoscopy in a critical setting. (J Thorac Cardiovasc Surg 2013;145:926-32)

During the past decade, substantial progress has been made in the development of new treatment options for patients with advanced lung cancer. $^{1,2}$ Ten years ago, most nonsurgical candidate lung cancer patients had the expectation of being treated with single-line chemotherapy plus concurrent, sequential, or palliative radiotherapy ${ }^{3}$ to prevent local progression and systemic disease or, at the minimum, to control symptoms. Currently, more strategic options and new drugs ${ }^{4}$ offer multiple lines of chemo-, maintenance, and target-oriented therapies with increasing success, tending to individualize lung cancer patient treatments. ${ }^{5,6}$ Despite all these therapeutic options, most of the tumors become uncontrollable, and a significant number of patients experience tumor-caused airway obstruction. ${ }^{7}$ Central airway involvement is a frequent occurrence in lung cancer patients. ${ }^{8}$ Furthermore, many other

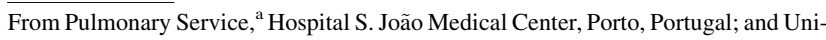
versity of Oporto Medical School, ${ }^{\mathrm{b}}$ Porto, Portugal.

Disclosures: Authors have nothing to disclose with regard to commercial support.

The investigation and all interventions were performed at Hospital S. João/Oporto Medical School.

Received for publication March 20, 2012; revisions received Aug 13, 2012; accepted for publication Aug 24, 2012; available ahead of print Oct 1, 2012.

Address for reprints: Venceslau Hespanhol, PhD, R. Silva Porto, n ${ }^{\circ} 418$ D 51, 4250470 Porto, Portugal (E-mail: vhespanhol@netcabo.pt).

$0022-5223 / \$ 36.00$

Copyright (c) 2013 by The American Association for Thoracic Surgery

http://dx.doi.org/10.1016/j.jtcvs.2012.08.066 cancer types metastasize to or invade the tracheobronchial tree by contiguity. ${ }^{7,9}$ Intense dyspnea, even life-threatening asphyxia, obstructive pneumonia, and hemoptysis are some of the usual complaints that bring these patients to a physician. The symptoms and signs depend on the characteristics of the tracheobronchial obstruction. ${ }^{10}$ Usually, after a period of unvalued mild to moderate signs and symptoms of airflow obstruction, a critical respiratory limitation is installed quickly, which leads to an immediate attempt to restore airflow to avert risk to the patient's life, regardless of the cause. ${ }^{9,10}$ In this situation, the decision-making process on whether to intervene is difficult both because of its urgency as well as the scarcity of clinical features and diagnostic means available to guide the bronchoscopy physician in each particular case. This study focuses on the decision making to perform therapeutic interventional bronchoscopy in patients with neoplastic disease of any etiology that causes severe central airway obstruction. We analyzed preoperative clinical parameters to determine their influence on the endoscopic outcome. A predictive model was constructed to assess the individual probability of endoscopic success.

\section{METHODS}

This was a retrospective study (approved by the Hospital S. João Ethics Committee, Institutional Review Board No. 31/2011). Electronic bronchoscopy records (filled out immediately at the end of the procedure) and the video endoscopic registry of patients who underwent rigid 
bronchoscopy under general anesthesia for treatment of severe neoplastic central airway obstruction from 1990 and 2009 were analyzed.

The great majority of the interventions were performed by the authors, as were the assessment and classification of events occurring before, during, and after endoscopic interventions. The study was done in the Pulmonary Department of the Hospital S. João, a 1000-bed university hospital in Porto, Portugal.

\section{Selection Criteria}

Patients were included after retrospective evaluation of electronic records of bronchoscopy performed with therapeutic intention from 1990 to 2009. Only cases with severe involvement of central airways (trachea, main carina, left and right main bronchus) were included. Any case with such an endoscopic neoplastic obstruction that could not be surpassed with a 4-mm Karl Storz telescope (Tullingen, Germany; at least $75 \%$ reduction of original tracheobronchial lumen) and did not allow visualization of the distal airway was deemed as severe involvement. ${ }^{11}$

\section{Evaluation Criteria}

Endoscopic treatment was classified as successful if, at the end of intervention, an $8.5-\mathrm{mm}$ Shapsay rigid bronchoscope (Tullingen, Germany; 10.5 -mm external diameter) could pass easily through the neoplastic obstruction and the airway lumen remains unchanged after bronchoscope removal (at least $50 \%$ improvement). The treatment was considered a failure when these conditions were not met.

\section{Preoperative Evaluation}

All patients were submitted previously to a judicious clinical evaluation by the pulmonologist responsible for endoscopic treatment. The evaluation included a clinical history and physical examination, complete blood count, blood chemistry, activating partial thromboplastin time, chest radiography or chest computed tomography, and, if possible, a review of preintervention endoscopic findings (fiberoptic bronchoscopy).

\section{Intervention}

Patients were intubated after anesthetic induction with an 8.5-mm Shapsay rigid bronchoscope tube (Karl Storz). The intravenous anesthesia protocol included prednisolone $1 \mathrm{mg} / \mathrm{kg}$, a bolus of propofol $2 \mathrm{mg} / \mathrm{kg}$, and succinylcholine $1 \mathrm{mg} / \mathrm{kg}$ immediately after anesthetic induction was established. Anesthetic maintenance was done using continuous propofol perfusion of $12 \mathrm{mg} / \mathrm{kg} / \mathrm{hour}$ during the first 10 minutes, $9 \mathrm{mg} / \mathrm{kg} / \mathrm{hour}$ from 10 to 30 minutes, and $6 \mathrm{mg} / \mathrm{kg} / \mathrm{hour}$ until the end of the intervention. More identical succinylcholine doses could be given at anytime according to muscle activity. Manual jet ventilation through the bronchoscope was used during the entire intervention. The neoplastic lesions (Figure 1) found inside the airways could assume 3 basic types: mass, defined as an intraluminal tumor; mucosal infiltration, characterized by vascular engorgement involving the mucosa and submucosa layers, edema, and irregularity of the mucosa caused by neoplastic cell invasion; and extrinsic compression, when luminal narrowing was caused by external pushing of the airways. More complex neoplastic involvement could be identified in the airways as a result of the association of these 3 basic lesions (Figure 1).

The operative strategy was decided case by case based on the preoperative evaluation. The endoscopic intervention involved instrumental manipulation with a bronchoscope, forceps, and an aspirator tube to drain smoke, blood, and small tissue debris, which is common to all types of treatment (instrumental debulking). A neodymium-yttrium aluminum garnet (Nd:YAG) laser (20-30 W, in pulses of 5-20 seconds; Laserscope, San Jose, Calif) was used to promote the coagulation and shrinkage of the endoluminal lesions. When the obstruction was determined by extrinsic compression or mucosal infiltration, stents (Dumon [Novatech, La Ciotat, France], Hood [Hood Laboratories, Pembrook, Mass]) were used. ${ }^{12,13}$ In cases of complex lesions, for instance, mass + extrinsic compression, laser therapy and mechanical debulking were performed to treat the endoluminal mass; a tracheobronchial stent was also considered to deal with extrinsic compression.

\section{Statistical Analysis}

The clinical and demographic characteristics of the patients (Table 1) were transformed into indicator variables, coded as 1 if the characteristic was present and as 0 if not. Gender was coded as 1 for male and as 2 for female. Age was expressed as median and age interval. A new dichotomous variable, age 75 , was coded as 1 if the patient was 75 years or older and as 0 if the patient was younger than 75 years. The endoscopic outcome was coded as 1 if considered a success and as 0 if regarded a failure. The interaction of all these variables with the interventional bronchoscopy outcome allowed obtaining a simple summary of their influence on the immediate endoscopic result, expressed as risk ratios (RRs). ${ }^{14} \mathrm{~A}$ logistic regression model was constructed ${ }^{14}$ to assess the association between the outcome (success/failure) and the preoperative patient characteristics. The logistic model we obtained ${ }^{14}$ was used to generate outcome estimates for each individual patient submitted to interventional bronchoscopy. The individual patient outcome estimates could be obtained by getting the sum of the products of the coefficient of each independent variable $(\beta 1-\mathrm{n})$ included in the logistic model (Table 2$)$ and their code $\left(\mathrm{X}_{1-\mathrm{n}}\right)-1$ if a particular characteristic was present and 0 if absent-and replacing this in the formula $\frac{1}{1+e^{-\left(\text {Constant }-\sum\left(\beta_{1} X_{1}+\beta_{2} X_{2}+\cdots \beta_{n} X_{n}\right)\right)}}$ to obtain the in dividual probability of endoscopic outcome (STATA Statistics Data Analysis 9.0, College Station, Tex). We used STATA Statistical Data Analysis 9.0 and BiostatXL MIX 2.0 (availabe at: http://www.meta-analysis-made-easy.com) to compute all these estimates.

\section{RESULTS}

We studied 804 patients during a 20 -year period (Table $1)$. The patients were primarily male $(\mathrm{n}=618,76.9 \%)$, and the median age was 62 years. Lung cancer was the most frequent neoplastic cause of airway obstruction $(\mathrm{n}=645,81.65 \%)$. Given the sudden presentation, the majority of the patients we studied underwent no more than a chest radiograph, blood count, biochemical study (ionogram, blood urea nitrogen, creatinine, and glucose), and summary endoscopic evaluation. Clinical clues, such as symptom exacerbation history, past radiographic images and computed tomographic scans, or initial endoscopic involvement, which would allow evaluation of how the disease began and how it evolved to the actual phase, were rarely available. This lack of information made it difficult to identify the tumor site of origin, which was of critical importance to bronchoscopy decision making and intervention outcome. Lobar atelectasis was the more often identified radiologic pattern on the patients we studied $(\mathrm{n}=341$, $43.27 \%$ ). Neoplastic airway involvement was observed in more than 1 site in 202 patients $(25.15 \%$; Table 1$)$.

An exophytic tumor was the most frequent single neoplastic lesion found in the airways of 436 patients $\left(54.23 \%\right.$; Table 1). The preoperative risk was established ${ }^{7}$ using the clinical evaluation and some complementary exams according to the individual patient's comorbidities.

The patient intervention risk assessment was performed after preoperative respiratory, cardiovascular, and hematologic clinical evaluation. ${ }^{7}$ Most of the patients we studied $(n=492$, $63.73 \%$ ) had an acceptable intervention risk. 

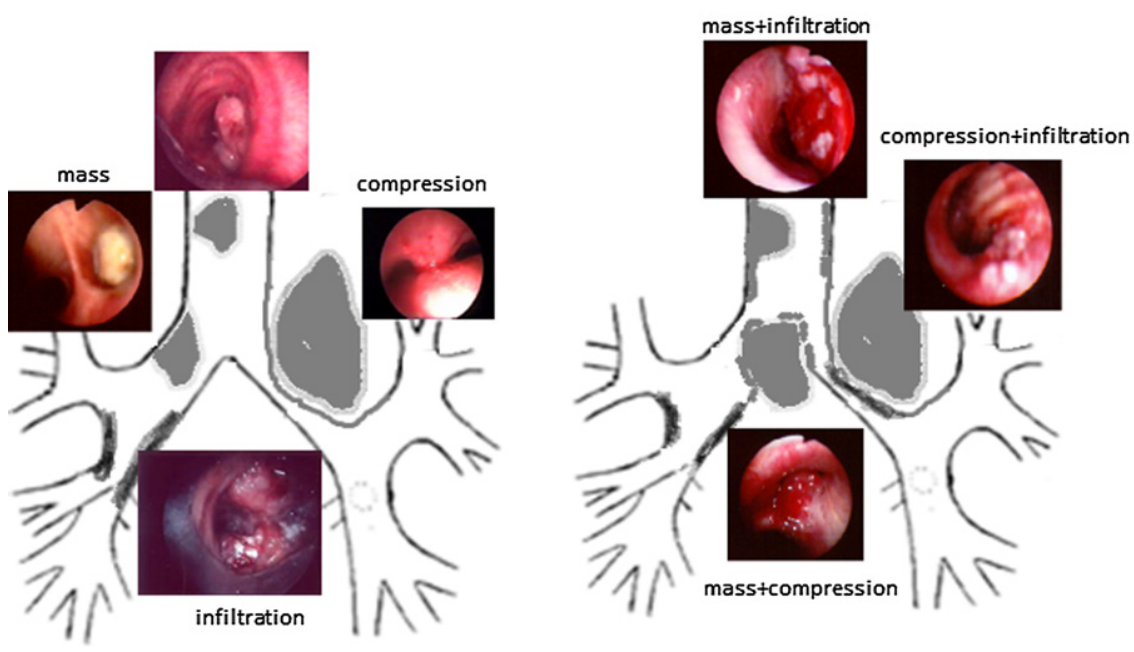

FIGURE 1. Endoscopic lesions: mass (intraluminal tumor), infiltration (vascular engorgement, edema, mucosal irregularity), compression (external tumor compression), and complex lesions (mass + infiltration, mass + compression, compression + infiltration).

Of the 804 patients with severe neoplastic obstruction of the airways submitted to endoscopic treatment, 681 $(84.7 \%)$ obtained luminal clearance of at least $50 \%$ of the original airway (Table 1), and their surgeries were considered endoscopic successes. The overwhelming majority of the patients who underwent interventional bronchoscopy had no adverse effects and recovered quickly and in a sustainable way, returning to their reference hospital within 3 hours after the procedure. The single perioperative death we had was caused by massive uncontrollable bleeding after a debulking attempt to clear the distal end of the left main bronchus (Table 2). Another death occurred as a result of cardiorespiratory arrest during anesthetic recovery.

Figure 2 shows the interaction between each patient characteristic and the bronchoscopy outcome. Female gender (RR, 1.07), tracheobronchial obstruction of esophageal origin (RR, 1.13), tracheal involvement (RR, 1.21), endobronchial mass (RR, 1.13), and extrinsic compression (RR, 1.17) were associated significantly with a favorable patient outcome.

The preintervention patient characteristics were used to construct a logistic model to predict the success or failure of performing a bronchoscopic therapeutic intervention to solve a severe neoplastic tracheobronchial obstruction. The model (Table 2) obtained points out 3 tumor localizations (tracheal, right main bronchus, and left main bronchus) and the presence of any kind of mucosal infiltration as the most important predictors of therapeutic bronchoscopic success.

Using these logistic estimates (Table 2), the probability of an individual patient with a severe malignant tracheal obstruction without endoscopic evidence of mucosal infiltration to get a good endoscopic result could be computed:

$$
\begin{aligned}
{[(1.25 \times 1)} & +(-1.38 \times 0)+(-0.63 \times 0)+(-2.44 \times 0) \\
& +(-1.44 \times 0)+(-1.14 \times 0)+2.65]=3.90,
\end{aligned}
$$

which indicates a probability of success of just less than $100 \%$ (Figure 3). On the other hand, a patient with left main bronchus obstruction by a mass and mucosal infiltration could have a much smaller probability of success:

$$
\begin{aligned}
{[(1.25 \times 0)} & +(-1.38 \times 1)+(-0.63 \times 0)+(-2.44 \times 0) \\
& +(-1.44 \times 1)+(-1.14 \times 0)+2.65]=-0.17,
\end{aligned}
$$

corresponding to an intervention success probability of less than $50 \%$ (Figure 3 ).

\section{DISCUSSION}

Lung cancer is the main cause of cancer death in the Portuguese male population. ${ }^{15}$ One of the most remarkable characteristics of a tumor spreading inside the airways is the way symptoms arise, many times in an abrupt and severe manner, after days or months of an overlooked mild evolution. This behavior demands a difficult prompt decision, taking into account the patient's complexity resulting not only from that particular tracheobronchial obstruction but also from performance status, comorbidities, and implication on life expectation. The challenge that this decision puts on such an emergency situation does not meet with accurate guidelines in the medical literature. ${ }^{16-21}$ Most of the published studies focus on the technical means, ${ }^{12}$ the strategy and different skills associated with the available treatments, ${ }^{16-19}$ the complications that may occur, ${ }^{9,10,12,22}$ and the outcomes and their influence on the prognosis of the disease. ${ }^{18}$ Despite the accumulated experience the surgeon may have, the decision to intervene in these patients remains a major challenge for many reasons other than the performance of the intervention itself. A miscalculation can result in a situation of no return.

To our knowledge, this is the first time a predictive model of success/failure in interventional bronchoscopy for severe 
TABLE 1. Patient data

\begin{tabular}{|c|c|c|c|c|}
\hline Characteristic (code) & No. of cases $(\%)$ & Success, n (\%) & Failure, n (\%) & Missing, $\mathbf{n}(\%)$ \\
\hline Hospital origin & & & & $60(7.50)$ \\
\hline H.S. João (1) & $314(42.20)$ & $266(84.71)$ & $48(15.29)$ & \\
\hline Other $(0)$ & $430(57.80)$ & $361(83.95)$ & $69(16.05)$ & \\
\hline \multicolumn{5}{|c|}{ Age, y (median, 26 y; range, 20-98 y) } \\
\hline Age category < 75 y (0) & $662(88.98)$ & $561(84.74)$ & $101(15.26)$ & $60(7.50)$ \\
\hline Age category $\geq 75$ y (1) & $82(11.02)$ & $67(81.71)$ & 15 (18.29) & 0 \\
\hline \multicolumn{5}{|l|}{ Sex } \\
\hline Male (1) & $618(76.87)$ & $515(83.33)$ & $103(16.67)$ & \\
\hline Female (2) & $186(23.13)$ & $166(89.25)$ & $20(10.75)$ & \\
\hline Tumor type $(*)$ & & & & $14(1.70)$ \\
\hline Lung $(1 / 0)$ & $645(81.65)$ & $537(83.26)$ & $108(16.74)$ & \\
\hline Thyroid (1/0) & $11(1.39)$ & $11(100.00)$ & $0(0.00)$ & \\
\hline Carcinoid (1/0) & $14(1.77)$ & $13(92.86)$ & $1(7.14)$ & \\
\hline Metastasis $(1 / 0)$ & $25(3.16)$ & $19(76.00)$ & $6(24.00)$ & \\
\hline Esophagus (1/0) & $70(8.86)$ & $66(94.29)$ & $4(5.71)$ & \\
\hline Other $(1 / 0)$ & $25(3.16)$ & $23(92.00)$ & $2(8.00)$ & \\
\hline Image (X-ray or CT scan) & & & & $16(1.90)$ \\
\hline Atelectasis (1) & $341(43.27)$ & $272(79.77)$ & $69(20.23)$ & \\
\hline Other (2) & $447(56.73)$ & $395(88.37)$ & $52(11.63)$ & \\
\hline Location $(*)$ & & & & $1(0.10)$ \\
\hline Trachea $(1 / 0)$ & $224(27.90)$ & $217(96.80)$ & $7(3.13)$ & \\
\hline Carina $(1 / 0)$ & $25(3.11)$ & $25(100.00)$ & 0 & \\
\hline RMB (1/0) & $173(21.54)$ & $145(83.82)$ & $28(16.18)$ & \\
\hline LMB $(1 / 0)$ & $202(25.15)$ & $171(84.65)$ & $31(15.34)$ & \\
\hline Multiple locations (1/0) & $179(22.29)$ & $122(68.16)$ & $57(31.84)$ & \\
\hline Endoscopic lesion $(*)$ & & & & 0 \\
\hline Mass & $436(54.23)$ & $390(89.45)$ & $46(10.55)$ & \\
\hline Extrinsic compression & $52(6.47)$ & $51(98.08)$ & $1(1.92)$ & \\
\hline Mucosal infiltration & $28(3.48)$ & $15(53.57)$ & $13(46.43)$ & \\
\hline Mass + compression & $80(9.95)$ & $73(91.25)$ & $7(8.75)$ & \\
\hline Mass + infiltration & $148(18.41)$ & $100(67.56)$ & $48(32.43)$ & \\
\hline Compression + infiltration & $60(7.46)$ & $52(86.66)$ & $8(13.34)$ & \\
\hline Intervention risk & & & & $32(3.80)$ \\
\hline Basal (0) & $492(63.73)$ & $412(83.74)$ & $80(16.26)$ & \\
\hline Risk (1) & $280(36.27)$ & $242(86.43)$ & $38(13.57)$ & \\
\hline Treatment $(*)$ & & & & 0 \\
\hline Laser $(1 / 0)$ & $598(75.51)$ & $485(81.10)$ & $113(18.90)$ & \\
\hline Stent $(1 / 0)$ & $129(16.28)$ & $126(97.67)$ & $3(2.33)$ & \\
\hline Laser + stent $(1 / 0)$ & $65(8.21)$ & $64(98.46)$ & $1(1.54)$ & \\
\hline Instrumental debulking $(1 / 0)$ & $12(1.48)$ & $10(83.33)$ & $2(16.67)$ & \\
\hline Outcome & & $681(84.70)$ & $123(15.30)$ & 0 \\
\hline
\end{tabular}

(*) Characteristics were transformed in indicator variables coded as 1 if the characteristic is present and 0 if it is not. $C T$, Computed tomography; $R M B$, right main bronchus; $L M B$, left main bronchus.

neoplastic central airway obstruction has been established. When we thought of doing this study, aimed at determining intervention criteria for performing therapeutic bronchoscopy in patients with respiratory distress caused by neoplastic involvement, many questions arose: Which strategy should we use? Which patients should be included? Which outcome would be acceptable to consider as an endoscopic therapeutic success? How could we assess accurately the outcome of the interventions?

A clinical trial would be unethical. A prospective study would be ideal; however, the number of accruable new cases each year (30-40) made it impossible to get results in due time. The only possibility lay in data from electronic bronchoscopy patient records started at our institution in 1988, which allowed us to perform this study, albeit retrospectively. Nevertheless, the information for each patient procedure was registered immediately after the bronchoscopy, thus minimizing the possible influence of information bias or misclassification of events measured at this distance in time. Another unique strength of the study lies in the fact that we performed the overwhelming majority of the interventions and hence their registry, thus reducing 
TABLE 2. Interventional bronchoscopy prediction model

\section{Logistic regression}

Correct model classification $84 \%$

\begin{tabular}{lcccccc}
\cline { 1 - 4 } \multicolumn{1}{c}{ Variables } & & & & & \multicolumn{2}{c}{ No. obs. 717 Prob. $>\boldsymbol{\chi}-\mathbf{0 . 0 0 0}$} \\
\cline { 1 - 6 } Trachea & Coefficient & Standard error & Wald & $\boldsymbol{P}$ value & Exp (coefficient) & Exp (coefficient) 95\% CI \\
Left main bronchus & 1.247 & 0.458 & 2.720 & .007 & 3.408 & $1.416-8.555$ \\
Right main bronchus & -1.377 & 0.285 & 23.257 & .000 & 0.252 & $0.144-0.442$ \\
Mucosal Infiltration & -0.626 & 0.318 & 3.884 & .049 & 0.535 & $0.287-0.997$ \\
Mass +infiltration & -2.441 & 0.475 & 26.435 & .000 & 0.087 & $0.034-0.221$ \\
Compression +infiltration & -1.442 & 0.255 & 31.899 & .000 & 0.236 & $0.143-0.390$ \\
Constant & -1.142 & 0.464 & 6.052 & .014 & 0.319 & $0.129-0.793$ \\
\hline
\end{tabular}

The location of the tumor and the morphology of the tracheobronchial lesions are determinants of the intervention success. Exp, Exponential; $C I$, confidence interval.

misinterpretation. The endoscopic outcome after an intervention bronchoscopy procedure is always difficult to quantify. The best solution we found was to determine $2 \mathrm{ob}-$ jective criteria that could be identified easily in patients' electronic records and video registry. Was the tracheobronchial obstruction overcome? At the end of the intervention, was at least $50 \%$ of the original lumen recovered? To consider a tracheobronchial lumen clearance of $50 \%$ as a good endoscopic result may be criticized. However, scientific knowledge on fluid flows through tubes allows us to recognize that any small clearance of a bronchus may correspond to an important gain in breathing efficiency that may, in fact, be clinically relevant.

The physiologic basis of this assumption is based on Poiseuille's law. ${ }^{23}$ According to this law, flow is related to perfusion pressure, radius, length, and viscosity. In the human body, however, flow does not conform exactly to this relationship. Nevertheless, the relationship shows clearly the dominant influence of radius on resistance and flow. This means that achieving even a small clearance of

\section{Bronchoscopy Outcome}

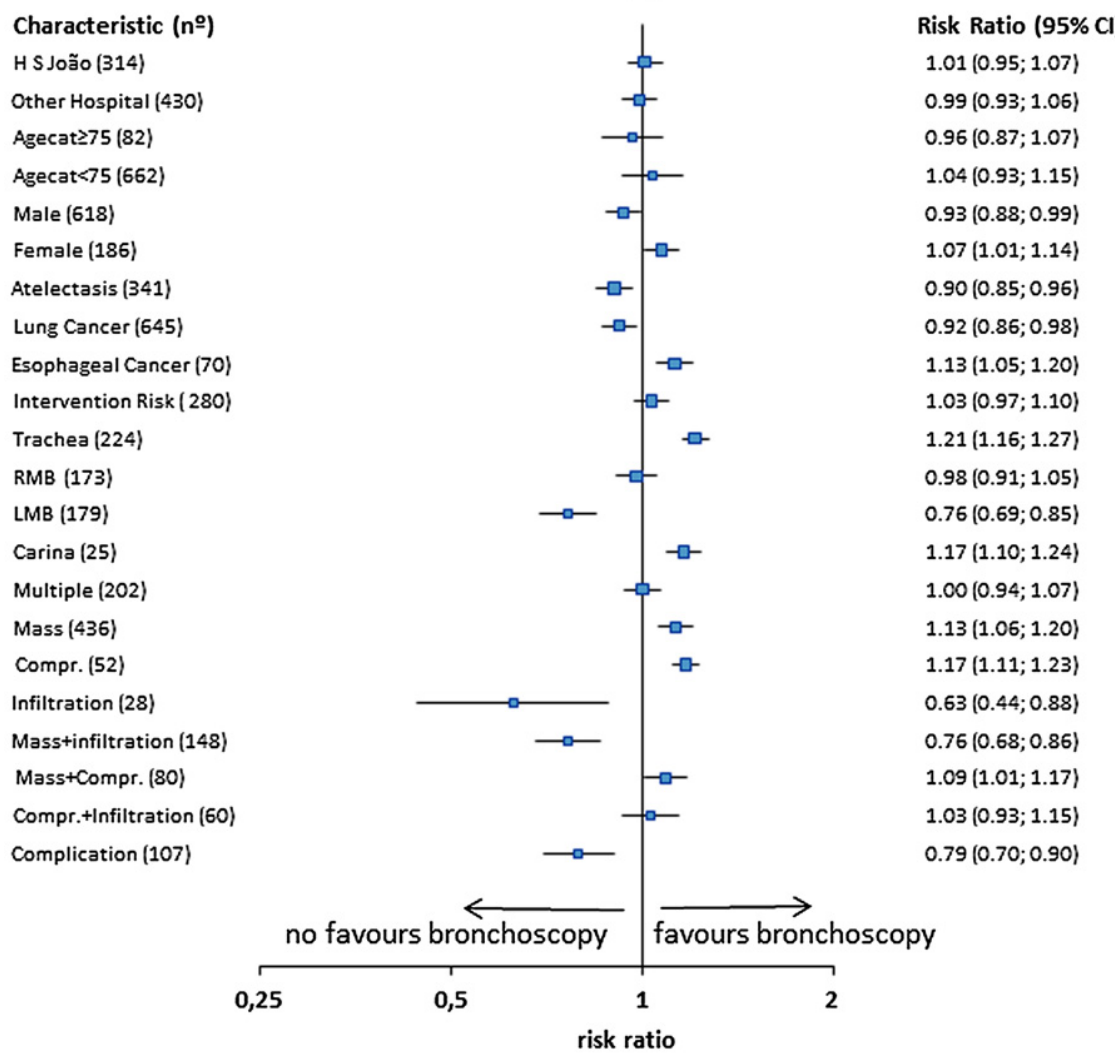

FIGURE 2. Interventional bronchoscopy group analysis. Risk ratios in performing bronchoscopy to treat severe tracheobronchial obstruction according to the presence of a particular patient characteristic. $R M B$, Right main bronchus; $L M B$, left main bronchus; $C I$, confidence interval. 


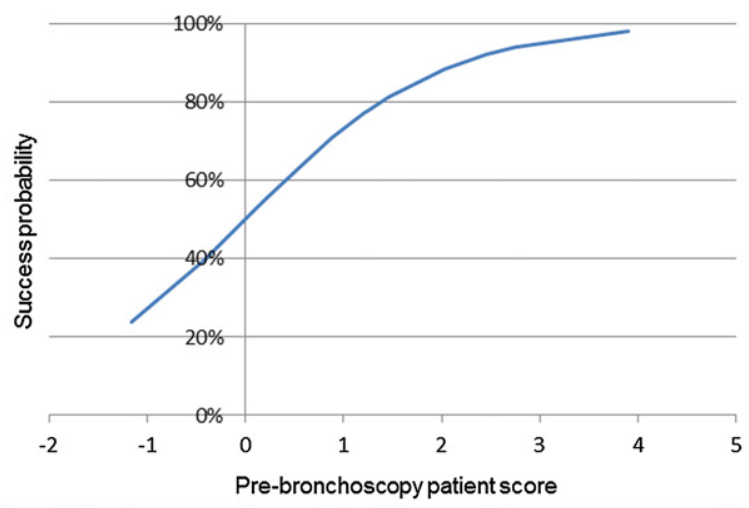

\begin{tabular}{|c|c|c|c|c|c|c|c|}
\hline Characteristic & No/Yes & $x$ & Coefficient & Score & Total Score & Prob. Success & Classification \\
\hline Trachea & $0 / 1$ & & 1.25 & & & & \\
\hline LMB & $0 / 1$ & & -1.34 & & \multirow[t]{2}{*}{$\geq 3$} & \multirow{2}{*}{$90-100 \%$} & \multirow{2}{*}{ Excellent } \\
\hline RMB & $0 / 1$ & & -0.63 & & & & \\
\hline Infiltration & $0 / 1$ & & -2.44 & & \multirow[t]{2}{*}{$\geq 2<3$} & \multirow[t]{2}{*}{$90 \%$} & \multirow[t]{2}{*}{ Good } \\
\hline Mass+Infiltration & $0 / 1$ & & -1.44 & & & & \\
\hline Compr+Infiltration & $0 / 1$ & & -1.14 & & $\geq 1<2$ & $75 \%$ & Fair \\
\hline Constant & & & 2.65 & & \multirow{2}{*}{$<1$} & \multirow{2}{*}{$50 \%$} & \multirow{2}{*}{ Poor } \\
\hline Total Score & & & & & & & \\
\hline
\end{tabular}

FIGURE 3. Interventional Bronchoscopy Success. Calculate the characteristic score by multiplying each logistic coefficient by 1 (if the characteristic is present) or by 0 (if the characteristic is absent). Sum all the characteristic scores to get the total score for an individual patient. Refer to the graph or the table to get patient's probability of endoscopic success. $R M B$, Right main bronchus; $L M B$, left main bronchus.

the bronchial lumen could decrease airflow resistance, which is clinically relevant.

One of the most significant limitations of this study is related to the patient selection done before the procedure. We submitted to interventional bronchoscopy only those patients who we were strongly convinced would have very good chances of benefiting from the treatment (patients with significant respiratory distress [extreme shortness of breath, polypnea, stridor, wheezing, and, sometimes, hypoxia], known severe malignant tracheobronchial obstruction based on fiber-optic bronchoscopy/computed tomographic scan, no other explanation for respiratory distress, and acceptable general condition performance status $0-3)$. Using these criteria, the patients presented here do not represent the entire spectrum of patients potentially approachable by interventional bronchoscopy, but rather those that, using preoperative clinical assessment, were classified as possible to benefit from this intervention. However, the model does seem to predict failure as well as success in the included patients, meaning worse cases would have been targeted easily by this statistical instrument. This predictive model points out the characteristics of the neoplastic lesion and the location of the obstruction as main determinants of intervention bronchoscopy success. The estimates provided by this model can be a very useful complement to the preintervention clinical evaluation toward making a decision on whether to perform interventional therapeutic bronchoscopy in patients with severe and complex conditions.

In conclusion, as mentioned, the tracheobronchial tumor location and the characteristics of the lesions are determinants of the endoscopic success of therapeutic interventional bronchoscopy. The use of the predictive rule proposed here might be a useful instrument, in addition to clinical evaluation, in increasing the accuracy of decision making on the performance of therapeutic bronchoscopy to treat severe neoplastic airway obstruction.

We thank Prof Paulo Dinis Oliveira for the final preparation of the manuscript, and bronchoscopy nurses Isilda Monteiro and Delfina Branco for their support during the interventions.

\section{References}

1. Stinchcombe TE, Hodgson L, Herndon JE II, Kelley MJ, Cicchetti MG Ramnath N, et al. Treatment outcomes of different prognostic group patients on Cancer Leukemia Group B trial 39801: induction chemotherapy compared with chemoradiotherapy alone for unresectable stage III non small cell lung cancer. J Thorac Oncol. 2009;9:1117-25.

2. Pao W, Girard N. New driver mutations in non small cell lung cancer. Lancet Oncol. 2011;2:175-80.

3. Schiller JH, Harrington D, Belani CP, Langer C, Sandler A, Krook J, et al. Comparison of four chemotherapy regimens for advanced non-small-cell lung cancer. N Engl J Med. 2002;346:92-8.

4. Mok TS, Wu IL, Thongprasert S, Yang CH, Chu DT, Saijo N, et al. Gefitinib or carboplatin plus paclitaxel in pulmonary adenocarcinoma. N Engl J Med. 2008; 361:947-57

5. Kwak EL, Bang YJ, Camidge DR, Shaw AT, Solomon B, Maki RG, et al. Anaplastic lymphoma kinase inhibition in non-small-cell lung cancer. $N$ Engl J Med. 2010;363:1693-703.

6. Cappuzzo F, Ciuleanu T, Stelmakh L, Cicenas S, Szczésna A, Juhász E, et al. Er lotinib as maintenance treatment in advanced non-small-cell lung cancer: a multicenter, randomized, placebo-controlled phase 3 study. Lancet Oncol. 2010;11: 521-9.

7. Drummond M, Magalhães A, Hespanhol V, Marques A. Rigid bronchoscopy: complications in a university hospital. J Bronchol. 2003;3:177-82.

8. Ernst A, Kopman DF, Becker HD, Mehta AC. Central airway obstruction. Am J Respir Crit Care Med. 2004;169:1278-97. 
9. Stephens KE Jr, Wood ED. Bronchoscopic management of central airways obstruction. J Thorac Cardiovasc Surg. 2000;119:289-96.

10. Jeon K, Kim H, Yu CM, Koh WJ, Suh GY, Chung MP, et al. Rigid bronchoscopic intervention in patients with respiratory failure caused by malignant central airway obstruction. J Thorac Oncol. 2006;4:319-23.

11. Brodsky JB, Macario A, Mark JBD. Tracheal diameter predicts double lumen tube size: a method for selecting left double lumen tubes. Anesth Analg. 1996;82:861-4.

12. Herth FJF. Endobronchial management of central cancers. Eur Respir Mon. 2009;44:336-48.

13. Hujala K, Sipilä J, Grenman R. Endotracheal and bronchial laser surgery in treatment of malign and benign lower airway obstruction. Eur Arch Otorhinolaryngol. 2003;260:219-22.

14. Harrell FE Jr, Lee KL, Mark DB. Multivariable prognostic models: issues in developing models, evaluating assumptions and adequacy, and measuring and reducing errors. Stat Med. 1996;15:361-87.

15. Ferlay J, Shin HR, Bray F, Forman D, Mathers C, Parkin DM. GLOBOCAN 2008 v1.2. Cancer incidence and mortality worldwide: IARC CancerBase No. 10. Lyon, France: International Agency for Research on Cancer; 2010. Available at: http://globocan.iarc.fr. Accessed January 15, 2011
16. Cavalieri S, Foccoli PO, Toninelli C, Feijo S. Nd:YAG laser in lung cancer: an 11year experience with 2235 applications in 1585 patients. J Bronchol. 1994;1: 105-11.

17. Wahidi MM, Heth FJF, Ernst A. State of the art: interventional pulmonology. Chest. 2007;131:262-74.

18. Brundage MD, Davies D, Mackillop WJ. Prognostic factors of non-small-cell lung cancer: a decade of progress. Chest. 2002;122:1037-57.

19. Murgu D, Colt GH. Interventional bronchoscopy from bench to bedside: new techniques for central and peripheral airway obstruction. Clin Chest Med. 2010;31:101-15.

20. Pyng L, Elif K, Atul M. Airway stent. Clin Chest Med. 2010;31:141-50.

21. Chhajed PN, Baty F, Pless M, Somandin S, Tamm M, Brutsche MH. Outcome of treated advanced non-small cell lung cancer with and without airway obstruction. Chest. 2006;130:1803-7.

22. Ernst A, Simoff M, Ost D, Goldman Y, Herth FJFl. Prospective risk-adjusted morbidity and mortality outcome analysis after therapeutic bronchoscopic procedures: results of a multi-institutional outcomes database. Chest. 2008;134: 514-9.

23. Pfitzner J. Poiseuille and his law. Anesthesia. 1976;2:273-5. 